\title{
VIOLÊNCIA FÍSICA CONTRA ENFERMEIROS ATUANTES NA CLASSIFICAÇÃO DE RISCO: OS DESAFIOS ENCONTRADOS NO AMBIENTE DE TRABALHO
}

\author{
PHYSICAL VIOLENCE AGAINST NURSES ACTING IN RISK \\ CLASSIFICATION: THE CHALLENGES FOUND IN THE WORKPLACE
}

\author{
Raimunda Idália Vieira Neta ${ }^{1}$ \\ Patrícia Pereira Tavares de Alcântara² \\ Rachel Cardoso de Almeida ${ }^{3}$ \\ Moziane Mendonça de Araújo ${ }^{4}$
}

Resumo: Objetivo: A presente pesquisa tem por objetivo identificar o conhecimento dos profissionais enfermeiros que atuam na classificação de risco sobre violência física no seu ambiente de trabalho, bem como conhecer a atitude desses profissionais frente a esse caso de violência. Método: Trata-se de uma pesquisa exploratória, descritiva e qualitativa, com enfermeiros de uma Unidade de Pronto Atendimento (UPA), em uma cidade localizada na região centro-sul do Ceará, no período de agosto e setembro de 2019, mediante a aplicação de um questionário semiestruturado, garantindo o sigilo e a privacidade dos participantes, respeitando a resolução 466/12, que trata sobre as pesquisas com seres humanos. Participaram do estudo oito enfermeiros. Resultados: Os dados foram analisados mediante as propostas de Minayo para pesquisas qualitativas e discutidos com literatura pertinente. Inicialmente realizou-se um diagnóstico socioeconômico dos participantes. Diante dos achados, pode-se perceber a tamanha dimensão e complexidade da violência física que os enfermeiros em Unidades de Pronto Atendimento vivenciam diariamente. A violência laboral é perceptível principalmente em serviços de urgência e emergência e sobretudo voltada aos profissionais de enfermagem em virtude da quantidade de tempo que permanecem com o paciente,

\footnotetext{
1 Enfermeira Graduada pela Universidade Regional do Cariri - URCA, Iguatu-CE. E-mail: idaliataiane@hotmail.com.

${ }^{2}$ Enfermeira. Docente no Curso de Graduação em Enfermagem da Universidade Regional do Cariri URCA. Mestre em Desenvolvimento Regional Sustentável pela Universidade Federal do Cariri UFCA. E-mail: enfermeira.tavares.81@gmail.com.

${ }^{3}$ Enfermeira. Docente no Curso de Graduação em Enfermagem da Universidade Regional do Cariri URCA. Especialista em Saúde da Mulher. E-mail: rachellcardosoo@gmail.com.

${ }^{4}$ Enfermeira. Docente no Curso de Graduação em Enfermagem da Universidade Regional do CaririURCA. Mestre em Enfermagem pela Universidade Federal do Ceará - UFC. E-mail: moziane@hotmail.com.
} 
bem como por realizarem a classificação de risco nesses serviços. Conclusão: Logo, percebeu-se que a violência no trabalho em saúde, e especificamente em enfermagem, vem se tornando um problema de saúde pública e que a violência ocupacional pode ser prejudicial à saúde e à carreira dos trabalhadores de enfermagem. E que, embora a sociedade venha sofrendo várias mudanças, os pensamentos e atitudes violentas estão cada vez mais presentes na nossa realidade.

Descritores: Enfermagem. Classificação de Risco. Violência Ocupacional.

Abstract: Objective: This research aims to identify the knowledge of professional nurses who work in the risk classification of physical violence in their work environment, as well as to know the attitude of these professionals towards this case of violence. Method: It was an exploratory, descriptive and qualitative study, with nurses from an Emergency Care Unit (UPA), in a city located in the south-central region of Ceará, in the period between August and September 2019, through the application of a semi-structured questionnaire, guaranteeing the confidentiality and privacy of the participants, respecting resolution 466/12 that deals with research with human beings. Eight nurses participated in the study. Results: The data were analyzed using Minayo's proposals for qualitative research and discussed with relevant literature. Initially, a socioeconomic diagnosis of the participants was carried out. In view of the findings, one can perceive the great dimension and complexity of physical violence that nurses in Emergency Care Units experience daily. Occupational violence is noticeable mainly in urgent and emergency services and, mainly, aimed at nursing professionals due to the amount of time they remain with the patient, as well as for performing the risk classification in these services. Conclusion: Soon, it was realized that violence at work in health and, specifically in nursing, has become a public health problem, and that occupational violence can be harmful to the health and career of nursing workers. And that, although society has undergone several changes, violent thoughts and attitudes are increasingly present in our reality.

Keywords: Nursing. Risk Classification. Occupational Violence. 


\section{INTRODUÇÃO}

Falar em saúde nos remete à história de como ocorreram os processos que culminaram no cenário atual. Dentre os maiores acontecimentos, tem-se o marco da realização da VIII Conferência Nacional de Saúde, em 1986, criando um amplo espaço à discussão sobre as principais propostas de assistência médica e saúde pública, sendo então proposto um arcabouço para a construção do que seria, em 1988, um grande avanço, a saber, que o direito à saúde constasse na Constituição da República de nosso país, base então para a criação do Sistema Único de Saúde (SUS) (BRASIL, 1988).

Com isso, foi instituído o SUS, mediante a promulgação da Lei 8.080 , de 1990, que dispõe sobre a promoção, proteção e recuperação da saúde, bem como a forma a qual o presente sistema deve ser organizado e, concomitantemente, a Lei 8.142, que dispõe sobre a participação dos indivíduos na construção do SUS (BRASIL, 1990a; BRASIL, 1990b).

No decorrer do tempo, os trabalhos no setor da saúde sempre tiveram significativa evolução e desenvolvimento, mesmo em cenários de crise econômica e desemprego, isso porque existe uma demanda que necessita destes profissionais, bem como por muitas das instituições serem sustentadas pelo Estado (GIRARDI; SEIXAS, 2002).

Para que haja, então, os serviços de saúde, é necessário o desenvolvimento do trabalho de várias categorias profissionais, entre elas o profissional de enfermagem. A enfermagem é uma profissão devidamente regulamentada no Brasil, mediante a Lei 7.498/86, (enfermeiros, técnicos de enfermagem, auxiliares de enfermagem e parteiras). Os profissionais de enfermagem estão cotidianamente ligados ao processo de cuidar, isso nos mais diversos setores de assistência à saúde, com o intuito de promovê-la e prevenir doenças e agravos de forma humanizada (BRASIL, 1986). 
Um dos locais de maior desafio em prestar esse tipo de atendimento é nos serviços de emergência, devido às grandes demandas. Os serviços de emergência, instituídos mediante a Portaria 2.048 do Ministério da Saúde (MS), vêm propor as unidades de atendimento de urgência e emergência e regulamentar que nessas unidades seja realizada a triagem com classificação de risco, por profissional de nível superior, seguindo um protocolo, objetivando avaliar as principais queixas que trouxeram esse usuário, ordenando as prioridades a serem atendidas da demanda que procura por esse serviço (BRASIL, 2009).

Sendo assim, o trabalho de triagem com classificação de risco é de fundamental importância, pois é a única forma de tentar organizar a demanda de acordo com a prioridade dos sintomas que os usuários apresentam ou verbalizam, classificando-os de acordo com o grau de prioridade em tempo que podem esperar pelo atendimento como emergente (cor vermelha: sem espera; até azul: duzentos e quarenta minutos). Essa triagem é uma atividade administrativa que pode ser realizada pelo enfermeiro ou médico (BRASIL, 2009).

Contudo, em meio a esse cenário onde se busca direcionar os pacientes a um atendimento igualitário e mais justo, deparamo-nos constantemente com os mais diversos tipos de violência contra os profissionais. Essa violência é a expressão no ato em si de violar o outro ou a si próprio. Para além disso, indica algo fora do habitual, ligado a força, e pode produzir danos como ferimentos, tortura e até mesmo a morte. Porém, o termo ainda aponta para violências psíquicas, verbais, ameaças, entre outros (MODENA, 2016).

O termo "violência" nos apresenta diversas definições. Contudo, Rocha (1996), apud, Almeida (2010) traz a violência como algo que vai além dos direitos humanos em se tratando de sua realidade e integridade física, social, psíquica, política e religiosa.

Segundo Silveira, et al. (2016), os mais variados tipos de violência aos quais os profissionais enfermeiros estão sujeitos na sua atividade laboral (violência verbal, psíquica e até mesmo física) trazem à discussão a necessidade de respeito por parte dos usuários, bem como de intervenções imediatas que venham a favorecer uma maior segurança no desenvolvimento de seu trabalho. 
Mediante o contexto abordado, surgiram os questionamentos que direcionaram este estudo: qual o conhecimento dos profissionais enfermeiros acerca da violência física? Já sofreram violência física no ambiente de trabalho? Como procedem diante de um caso em que sejam vítimas de violência física?

Justifica-se a pesquisa em função da necessidade de reconhecer as nuances da violência física a qual os profissionais enfermeiros estão sujeitos, sobretudo durante o exercício de sua atividade laboral em serviços de urgência/emergência para a realização do acolhimento com classificação de risco.

O interesse por essa temática surgiu da vivência da pesquisadora enquanto profissional de saúde (técnica de enfermagem), que instigou seu desejo de elucidar questões que permeiam o ambiente de trabalho da enfermagem.

O debate possui relevância por acreditar-se que, através do conhecimento proporcionado, possa haver mudança na realidade encontrada. Ressaltando-se que a presente temática já foi abordada na Agenda Nacional de Prioridades de Pesquisa em Saúde (ANPPS), a saber, a necessidade de realizar pesquisas em torno da avaliação dos sistemas de vigilância de violências e acidentes (incluindo os que ocorrerem no exercício do trabalho) (BRASIL, 2015).

Logo, o presente estudo se propõe a identificar o conhecimento dos profissionais enfermeiros que atuam na classificação de risco sobre violência física no seu ambiente de trabalho, bem como conhecer a atitude desses profissionais frente a esse caso de violência.

\section{MÉTODOS}

\section{Tipo de Estudo}

A presente pesquisa teve caráter descritivo-exploratório e de abordagem qualitativa. 
Ocorreu de forma descritiva, considerando que Prodanov \& Freitas (2013) nos apresentam que nesse tipo de pesquisa, o pesquisador deve observar e registrar os dados analisados sem intervir em momento algum sobre a veracidade dos mesmos.

Assumiu caráter exploratório visto que procurará desenvolver, esclarecer e modificar conceitos e ideias, tendo em vista a formulação de problemas mais precisos ou hipóteses pesquisáveis para estudos posteriores (GIL, 2016).

Quanto à abordagem, foi qualitativa, visto que ela se desenvolve numa situação natural, oferecendo riqueza de dados descritivos, além de focalizar na realidade de forma complexa e contextualizada. Sendo assim, foram descritos os dados obtidos de forma que podemos em suas linhas perceber a subjetividade expressa por cada entrevistado, enquanto alguém imerso em um ambiente de trabalho que possa oferecer riscos a sua saúde durante o desenvolvimento de suas atividades laborais (LAKATOS; MARCONI, 2017).

\section{Cenário da Pesquisa}

O estudo foi desenvolvido na cidade de Iguatu, cidade localizada na região centro-sul do Ceará, distante aproximadamente $377 \mathrm{Km}$ da capital do estado (Fortaleza) (IBGE, 2017).

Ressalta-se que o município em questão possui uma rede bastante ampla de serviços de saúde, oferecendo serviços nos presentes estabelecimentos: Unidade de Pronto Atendimento (UPA), Hospital Regional de Iguatu (HRI), Centro de Especialidades Odontológicas (CEO), Estratégias de Saúde da Família (ESF) (sendo 12 na zona urbana e 18 na zona rural), entre vários outros (IGUATU, 2016).

O local onde ocorreu a pesquisa foi a Unidade de Pronto Atendimento (UPA) da cidade mencionada, sendo essa de porte I, com capacidade para realizar 150 atendimentos por dia, atendendo uma média mensal de 4.500 pacientes. A equipe multiprofissional é composta por dois médicos e dois enfermeiros que trabalham na assistência e classificação de risco, três técnicos de enfermagem e oito profissionais entre serviço social, maqueiros, atendentes, vigias, entre outros. 
A pesquisa foi realizada entre os meses de agosto e setembro de 2019.

\section{Participantes da Pesquisa}

Os participantes da pesquisa foram os oito enfermeiros que desempenham suas atividades laborais no serviço de atendimento de Urgência e Emergência com sistema de Classificação de Risco, da UPA da referida cidade.

Como critérios de inclusão, foram considerados os enfermeiros que desempenham suas atividades laborais no serviço de saúde supracitado e que utilizam em sua prática laboral o atendimento com estratificação de risco. Como critérios de exclusão, foram considerados os enfermeiros que se encontrarem de férias, licença maternidade, licença médica, entre outros.

\section{Técnicas e Instrumento de Coleta de Dados}

Para levantamento de dados a serem discutidos, foi realizada uma entrevista semiestruturada contendo perguntas abertas que possibilitaram ao entrevistado discorrer sobre questionamentos que envolvem os objetivos da pesquisa.

Vale ressaltar ainda que a escolha pela entrevista semiestruturada vai de encontro às contribuições de Manzini (2012) e Oliveira (2011), que nos apresentam sua importância nas pesquisas qualitativas, uma vez que dão ao pesquisador a flexibilidade em alterar a ordem das perguntas para ir de encontro aos objetivos da pesquisa, possibilitando a captação do que o entrevistado sabe, bem como o que deseja expressar em torno do que vem sendo discutido.

Para tanto, o roteiro de entrevista foi adaptado, seguindo as premissas de Bordigon e Monteiro (2015), que desenvolveram um questionário a nível nacional para o levantamento de dados sobre os casos de violência com profissionais de saúde, com foco nos profissionais de enfermagem, dada a alta prevalência de serem 
acometidos, tendo então a possibilidade de fazer melhor mensuração sobre seus casos e como esses têm ocorrido.

A coleta de dados sucedeu mediante encontro agendado, respeitando a prévia disponibilidade do participante, no próprio ambiente de trabalho, em dias úteis da semana, em sala confortável, assegurando sigilo e confidencialidade das informações.

As falas foram gravadas e transcritas na íntegra, para que nenhuma informação fosse perdida e para que possibilitasse melhor interpretação do diálogo, assegurasse a fidedignidade das informações e enriquecesse a coleta dos dados. $E$ logo após transcritas foram apagadas.

\section{Organização e Análise dos Dados}

Os dados foram devidamente organizados de acordo com a temática a ser analisada e discutida. Para isso lançamos mão da técnica de análise de conteúdo proposta por Minayo (2014).

A organização do material ocorreu mediante a leitura e interpretação legítima das contribuições dos sujeitos em estudo, e transcrição das falas, formando então as categorias temáticas que utilizamos posteriormente para confrontar com a literatura sobre a temática em questão (MINAYO, 2012).

Por fim, a análise temática foi dividida em três momentos distintos, sendo cada um de extrema importância para que o seguinte ocorra de forma satisfatória. Primeiramente, a pré-análise (caracterizada pela organização do material que foi analisado), por conseguinte a exploração (onde o autor fez as devidas codificações na transformação do texto de linguagem simplificada para linguagem científica) e, por fim, o terceiro momento, que será a abordagem dos resultados obtidos e interpretados, ou seja, o trabalho que o autor fez com os resultados com os quais se deparou em campo, possibilitando destacar e interpretar as informações obtidas (MINAYO et al, 2011). 


\section{Aspectos Éticos e Legais}

Com fins de garantir a integridade, segurança e direitos dos participantes desta pesquisa, tomou-se como base os preceitos estabelecidos pela Resolução 466/12, do Conselho Nacional de Saúde (CNS), que trata de pesquisas realizadas com seres humanos, embasados nos princípios de bioética, garantindo assim os direitos e deveres condizentes aos participantes da pesquisa na comunidade científica (BRASIL, 2013).

Inicialmente, foi solicitada anuência para a realização da pesquisa junto à Escola de Saúde Pública de Iguatu (ESPI), visto ser órgão municipal outorgado à autorização de pesquisas nesse município. Após parecer favorável à realização da pesquisa, o termo de anuência foi enviado à Plataforma Brasil para apreciação do Comitê de Ética em Pesquisa (CEP) da Universidade Regional do Cariri, obtendo a aprovação mediante parecer número 3.606.678.

Sobre a participação do público-alvo na pesquisa, estes foram esclarecidos de forma verbal sobre os objetivos da pesquisa, os procedimentos, bem como sobre os riscos e benefícios e, mediante a leitura do Termo de Consentimento Livre e Esclarecido (TCLE), e após conhecimento da pesquisa e todos os pontos ora expostos, assinaram o Termo de Consentimento Pós-Esclarecido (TCPE), em duas vias, ficando uma com o entrevistado e outra com o pesquisador.

O anonimato dos participantes desta pesquisa permaneceu garantido pelo uso de pseudônimos, sendo atribuído aos participantes nomes de pássaros (BeijaFlor, Araponga, Seriema) durante a apresentação dos resultados.

A pesquisa apresentou riscos mínimos relacionados ao constrangimento apresentado em responder o Roteiro de Entrevista. Tendo em vista a minimização desses riscos, a pesquisadora realizou a coleta de dados em ambiente silencioso e confortável, assegurando a garantia de sigilo de todos os dados, inclusive de informações pessoais e íntimas.

Contudo, vale enfatizar que os benefícios oferecidos pela pesquisa superaram os riscos, pois buscou contribuir com a ampliação de discussões acerca da violência 
laboral à qual os enfermeiros estão expostos durante a realização do trabalho de classificação de risco nos serviços de urgência e emergência.

\title{
RESULTADOS E DISCUSSÃO
}

Quando questionados sobre os aspectos essenciais para delinear o grau de conhecimento dos profissionais a respeito da violência física presente no desempenho das suas atividades laborais correlacionando ao fato de atuarem diretamente na estratificação de risco, as falas ilustram:

\begin{abstract}
"Violência física eu entendo que é quando se usa a força de forma intencional ou contra si mesmo ou contra outra pessoa com o objetivo de machucar, de ferir, de causar algum dano físico a essa pessoa" (Beija-flor).

"O maior problema que se tem na classificação de risco se dá pelo pessoal querer ser atendido na frente dos outros, da questão da demora, ninguém quer aguardar, o pessoal não tem empatia com o próximo de ter a preocupação de saber se aquele problema do outro é maior ou menos do que o dele, ele quer saber se passa na frente, tem pessoas que às vezes está enfartando lá dentro e a pessoa quer ser atendido na frente" (Curió).
\end{abstract}

Observou-se nas respostas dos prticipantes que eles conseguem definir de maneira singular e objetiva a violência física. Um dos entrevistados ainda aponta uma possível barreira no desenvolvimento da estratificação de risco em detrimento de ações comportamentais apresentadas pelos pacientes que aguardam 0 atendimento.

No que diz respeito ao acolhimento e classificação de risco, segundo Bellucci e Matsuda (2012), o acolhimento ao usuário pode ser realizado por qualquer profissional de saúde desde que esse seja habilitado para esse fim; porém, a classificação de risco é de competência do enfermeiro e é realizada durante a 
primeira etapa do Processo de Enfermagem, apresentando a queixa principal do usuário e, diante de um protocolo preestabelecido na unidade, classificando-o em um sistema de cores para sua urgência de saúde.

Tal classificação pode gerar descontentamento ao paciente, acarretando em violência. Di Martino (2003) ainda traz que, ao analisar quanto à experiência de enfermeiros com a violência relacionada ao paciente e visitante em ambientes hospitalares, pesquisas na área mostram que 95\% dos enfermeiros vivenciaram esse incidente durante a carreira, $72 \%$ experimentaram o abuso verbal e $42 \%$ a violência física. Os profissionais de enfermagem e também os demais da saúde estão sujeitos a esses eventos, por serem considerados comuns entre os trabalhadores que estabelecem contato com pessoas em situações de angústia.

Quando interrogados a respeito de algum episódio de violência física no seu labor, obtivemos as seguintes respostas:

"Já! Na verdade foi quando um paciente estava aguardando, e com a demora sofri empurrões, foi apenas empurrão não chegou a bater, não!” (Tucano).

"Não! Nunca sofri nenhum tipo de violência física" (Uirapuru).

Verificou-se pela percepção da pesquisadora que um entrevistado demonstrou incômodo ao relembrar a ocorrência do fato sofrido, por ter uma experiência marcante. Porém, os demais entrevistados do estudo relataram que nunca experienciaram um fato de violência física no setor de trabalho.

A palavra "violência" vem do termo em latim "vis", que significa força, numa perspectiva de constrangimento, uso da força física sobre o outro, em uma relação de desigualdade. Há violências que são aceitas entre os indivíduos, porém, outras não. Em todos os espaços que o homem ocupou, a violência se fez presente, mudando só a forma como se apresentava (CASIQUE; FUREGATO, 2006).

Em vista disso, no final da década de 1980, a violência no trabalho passou a ser adotada como uma questão relacionada para a saúde dos trabalhadores. Estudos apontam que, no ano supracitado, dentre as mortes com causas associadas ao trabalho, entre $12 \%$ e $17 \%$ incidiram em homicídios (TOSCANO; WEBER, 1995). 
Dessa forma, pesquisas apontam que as mortes sucedidas por violência no trabalho apresentam como vítima principalmente homens, trabalhadores do setor de vendas a varejo, transporte urbano, bares e restaurantes, e se relacionam a assaltos e roubos. De outro modo, violências não fatais referem-se em primeiro lugar às mulheres, profissionais dos serviços de saúde, principalmente o enfermeiro, e tem como causador, em geral, um paciente que adentra a unidade de cuidado (CAMPOS; PIERANTONI, 2010).

Assim, observa-se que os serviços de saúde onde as ocorrências de violência física ou verbal entre trabalhadores, clientes ou usuários são mais frequentes, possuem regras e formas de funcionamento próprias, por exemplo, podem produzir insatisfação, frustração e sentimento de injustiça por parte dos usuários. Com isso, os trabalhadores tornam-se vulneráveis às reações agressivas por parte desses usuários, visto que os trabalhadores são atores das regras dos serviços ou protocolos existentes (CAVALCANTE et al., 2013).

Os participantes do estudo ainda foram interrogados sobre terem vivenciado algum problema decorrente do uso da classificação de risco. Eles relataram em sua maioria que sim, e que diariamente encaram diversas situações difíceis. As falas abaixo ilustram:

"Sim! Porque às vezes as pessoas não querem respeitar a classificação de risco, acham que estão mais graves do que outras pessoas que estão "lá fora", ou acham que porque é o mais velho, tem que entrar na frente..." (Araponga).

“Já, justamente esta agressão foi por conta da classificação, que estava demorando um pouco e o paciente se irritou muito" (Tucano).

"Sim! Como a gente trabalha em um ambiente de urgência e emergência a classificação de risco ela se propõe justamente para organizar o fluxo do atendimento." (Uirapuru).

Diante disso, é observado um cenário preocupante, uma vez que os profissionais estão em seu local de trabalho, exercendo seu papel de cuidador, e 
ainda assim, necessitem passar por situações adversas ou situações de violência física ou verbal por parte dos usuários.

Os profissionais de saúde que sofrem com diversas formas de violência devem contar com orientação de programas de apoio na resolução de seus problemas, sejam psicológicos, físicos, familiares e até profissionais, tanto governamentais quanto não governamentais, ficando evidente que a violência sofrida no ambiente laboral tem se transformado numa forma cada vez mais brutal de violência contra o profissional, mesmo que ele já possa contar com atendimento especializado (SILVA, 2005).

O enfermeiro é sujeito fundamental no funcionamento eficiente da classificação de risco é responsabilidade específica e competente do enfermeiro. Este quem concretiza o julgamento clínico e crítico das queixas recebidas e, a partir dessas informações colhidas, determinará o risco para cada caso específico. Portanto, para esse fim, o enfermeiro deve avaliar todas as etapas do processo de maneira holística e humanizada; além disso, ele deve observar a abrangência do Acolhimento com Classificação de Risco (ACCR), desmistificando o conceito de que se trata apenas de um local ou parte do atendimento da unidade (ACOSTA; DURO; LIMA, 2012).

Nesse sentido, vários países passaram a adotar a classificação de risco como protocolo de atendimento aos seus usuários, pois ela é testada e adequada para cada região do país. O Brasil, através do Sistema Único de Saúde (SUS), vem ampliando novas propostas no sentido de melhorar a organização do atendimento na rede de atenção às urgências e emergências (REGINA et al., 2009).

Corroborando com o explicitado anteriormente, os entrevistados foram questionados quanto ao que fizeram após a ocorrência de alguma violência física. Os que experimentaram essa ocorrência argumentaram ter anotado no livro de ocorrências da unidade e/ou procurado um local de registros de Boletim de Ocorrências (B.O) para uma ocorrência prestada.

"Só registrei no prontuário, porque o paciente estava em surto! Não podia fazer nada contra ele não" (Araponga).

“Eu fui na delegacia fazer um B.O” (Tucano). 
Baptista (2017) traz que a violência nos locais de trabalho vem somando de maneira drástica. Isso não é um problema somente da Enfermagem. É de qualquer serviço prestado à sociedade, tornando-se uma realidade global ocorrida tanto em países já desenvolvidos como em desenvolvimento e em vários tipos de trabalho e grupos ocupacionais.

Com isso, a pesquisa nacional que investigou o uso de psicofármacos pelos trabalhadores de Enfermagem confirmou que $21,7 \%$ dos trabalhadores que sofrem/sofreram violências fazem uso dessas medicações, com maior consumo entre profissionais que atuam em Unidades de Terapia Intensiva - UTI (34,78\%) e Unidades de Internação - UI (34,78\%), e máxima prevalência no consumo de psicofármacos entre técnicos de Enfermagem (22,8\%), sendo os fundamentais motivos a depressão, ansiedade e insônia (VASCONCELLOS; GRIEP; LISBOA et al., 2015).

Portanto, quando se afeta a saúde dos trabalhadores, a violência no trabalho impacta no gerenciamento de recursos humanos em Enfermagem e, possivelmente, no resultado final do serviço prestado. A violência tem repercussões para o indivíduo, para o coletivo e para a instituição. Desse modo, torna-se importante adotar medidas que protejam os profissionais de atos violentos.

\section{CONCLUSÕES}

Diante da presente pesquisa, percebeu-se a dimensão e complexidade da violência física que os enfermeiros em Unidades de Pronto Atendimento vivenciam diariamente. E que, embora seja considerada um grave problema de saúde pública, apresenta-se permeada de tabus. Para a sociedade, falar sobre essa temática é expor o ambiente de trabalho, os profissionais de saúde e os usuários.

$O$ estudo permitiu reconhecer os limites e as potencialidades da prática assistencial das UPAs, bem como subsidiar reflexões para o planejamento de ações que transformem e fortaleçam as fragilidades evidenciadas. 
A pesquisa não apresentou limitações para sua realização; porém, observouse uma certa resistência por parte de alguns participantes durante momentos da coleta; contudo, isso não interveio no progresso da entrevista.

Ao longo do estudo, observou-se a importância do enfermeiro na aplicação da classificação de risco, sendo esse profissional qualificado direcionado à avaliação integral do paciente e não apenas ao diagnóstico, verificando que, mesmo diante dos entraves vivenciados, consegue desenvolver suas atividades laborais com qualidade.

Logo, o presente estudo reforça a necessidade de novas pesquisas relacionadas ao profissional enfermeiro no Brasil, cujo papel como profissional responsável pela triagem com acolhimento e classificação de risco não é valorizado repercutindo assim no estresse que atrapalha o desempenho dos profissionais.

\section{REFERÊNCIAS BIBLIOGRÁFICAS}

ACOSTA, A.; DURO, C.; LIMA, M. Atividades do enfermeiro nos sistemas de triagem/classificação de risco nos serviços de urgência: revisão integrativa. Revista Gaúcha de Enfermagem, p. 181-190, 2012.

BAPTISTA, P.C.P. Violência no trabalho: guia de prevenção para profissionais de enfermagem. ISBN: 978-85-68720-08-0. São Paulo: Coren-SP, 2017.

BELLUCCI, J. J. A; MATSUDA, L. M. Implantação do acolhimento com classificação de risco em serviço hospitalar de emergência: atuação do enfermeiro. Ciênc Cuid Saúde. 2012; 11(2):396401.

BORDIGNON, M.; MONTEIRO, M. I. Validade aparente de um questionário para avaliação da violência no trabalho. Acta Paulista de Enfermagem, São Paulo, v. 6, n. 28, p.601-608, out. 2015. Disponível em: <http://www.scielo.br/pdf/ape/v28n6/1982-0194-ape-28-06-0601.pdf>. Acesso em: 04 abr. 2019.

BRASIL. Constituição da República Federativa do Brasil (1988). Brasília-DF. Diário Oficial da República Federativa do Brasil, $1988 . \quad$ Disponível em: http://www.planalto.gov.br/ccivil_03/constituicao/constituicao.htm. Acesso em: 17 Abr. 2019.

Departamento de Ciência e Tecnologia. Ministério da Saúde. Agenda Nacional de Prioridades de Pesquisa em Saúde. 2. ed. Brasília: Editora do Ministério da Saúde, 2015. Disponivel em: http://brasil.evipnet.org/wp-content/uploads/2017/07/ANPPS.pdf. Acesso em: 17 Abr. 2019.

. Lei № 8080, de 19 de Setembro de 1990. Dispõe sobre as condições para a promoção, proteção e recuperação da saúde, a organização e o funcionamento dos serviços correspondentes e dá outras providências. Brasília-DF, 19 de Setembro de 1990. Diário Oficial
da
República
Federativa
do
Brasil.
Disponível
em:

http://www.planalto.gov.br/ccivil_03/leis/L8080.htm. DFY Acesso em: 17 Abr. 2019 
Lei № 8142, de 28 de Dezembro de 1990. Dispõe sobre a participação da comunidade na gestão do Sistema Único de Saúde (SUS) e sobre as transferências intragovernamentais de recursos financeiros na área da saúde e dá outras providências. Brasília-DF, 28 de Dezembro de 1990. Diário Oficial da República Federativa do Brasil. Disponível em: http://www.planalto.gov.br/ccivil_03/ leis/_L8142.htm. Acesso em: 17 Abr. 2019.

. Lei $N^{0} 7.498$, de 25 de Junho de 1986. Dispõe sobre o exercício da enfermagem, e dá outras providências. Brasília-DF, 26 de Junho de 1986. Diário Oficial da República Federativa do Brasil. Disponível em: http://www.planalto.gov.br/ccivil_03/LEIS/L7498.htm. Acesso em: 17 Abr. 2019.

Ministério da Saúde. Secretária de Atenção à Saúde. Acolhimento e classificação de risco nos serviços de urgência. Brasília: Ministério da Saúde, 2009. 59 p. Disponível em: http://bvsms.saude.gov.br/bvs/publicacoes/acolhimento_classificaao_risco_servico_urgencia.pdf. Acesso em: 17 Abr. 2019.

CASIQUE, L. C.; FUREGATO, A. R. F. Violência Contra Mulheres: reflexões teóricas. Rev Latino-am Enfermagem, 2006 novembro-dezembro; 14(6). www.eerp.usp.br/rlae. Artigo de Revisão Online.

CAMPOS, A.S.; PIERANTONI, C. R. Violência no trabalho em saúde: um tema para a cooperação internacional em recursos humanos para a saúde. Rev. Eletr. De Com. Inf. Inov. Saúde. Rio de Janeiro, v. 4, n.1, p. 86-92, mar.2010.

CAVALCANTE, R. et al. Acolhimento com Classificação de Risco: proposta de humanização nos serviços de urgência. Revista de Enfermagem do Centro-Oeste Mineiro, 2013.

DI MARTINO V. Relationship between work stress and workplace violence in the health sector. Geneva; 2003. 33p.

GIL, A. C. Como elaborar projetos de pesquisa. $6^{a}$. Ed. São Paulo: Atlas, 2016.

GIRARDI, S. N.; SEIXAS, P. H. Dilemas da regulamentação da área da saúde: questões para um governo democrático e exclusista. Revista Latinoamericana de Estudios del Trabajo, Buenos Aires, n. 5, Maio, 2002. Disponível em: http://bvsms.saude.gov.br/bvs/is_digital/is_0203/pdfs/IS23(2)051.pdf. Acesso: em 20 Abr. 2019.

IBGE. Instituto Brasileiro de Geografia e Estatística. Sinopse do Censo Demográfico 2010 Brasil. 2014. Disponível em: <http://www.ibge.gov.br/home/estatistica/populacao/censo2010/>. Acesso em: 17 Abr. 2019.

IGUATU. Secretaria Municipal de Saúde. Plano municipal de Saúde 2012/2016. Iguatu: Secretaria Municipal de Saúde, 2016. Disponível em: <http://iguatu.ce.gov.br/secretaria-desaude-fms/>. Acesso em: 17 Abr. 2019.

LAKATOS, E. M.; MARCONI, M. de A. Metodologia Científica. 7. Ed. São Paulo: Atlas, 2017.

MANZINI, E. J. Uso da entrevista em dissertações e teses produzidas em um programa de pósgraduação em educação. Revista Percurso, Maringá, v. 4, n. 2, p.149-171, 2012. Disponível em: <http://periodicos.uem.br/ojs/index.php/Percurso/article/view/18577/10219>. Acesso em: 17 Abr. 2019

MINAYO, M. C. S. O desafio do conhecimento: pesquisa qualitativa em saúde. $14^{a}$ ed. Editora Hucitec. São Paulo, 2014.

MINAYO, M. C. S. Análise qualitativa: teoria, passos e fidedignidade. Ciência \& Saúde Coletiva, Rio de Janeiro, v. 17, p.621-626, 2012. 
MINAYO, M. C. de S.; DESLANDES, S. F.; GOMES, R. (Org.). Pesquisa social: teoria, método e criatividade. In: (ORG.), Maria Cecília de Souza Minayo; DESLANDES, Suely Ferreira; GOMES, Romeu. 30. ed. São Paulo: Petrópolis, 2011.

MODENA, M. R. et al. Conceitos e formas de violência. Caxias do Sul: Editora da Universidade de Caxias do Sul, 2016. 69 p. Disponível em: $<$ https://www.ucs.br/site/midia/arquivos/ebook-conceitos-formas_2.pdf>. Acesso em: 11 jul. 2018.

OLIVEIRA, M. F. Metodologia Científica: um manual para a realização de pesquisas em administração. $2011 . \quad$ Disponível em: <https://adm.catalao.ufg.br/up/567/o/Manual_de_metodologia_cientifica_-_Prof_Maxwell.pdf>. Acesso em: 10 Mai. 2019.

PRODANOV, C. C.; FREITAS, E. C. de. Metodologia do trabalho científico: Métodos e técnicas da pesquisa e do trabalho acadêmico. $2^{\mathrm{a}}$. Ed. Novo Hamburgo: Feevale, 2013.

REGINA, E. G. et al. Organização do trabalho de uma equipe de saúde no atendimento ao usuário em situações de urgência e emergência. Texto e Contexto - Enfermagem, v. 18, 2009.

SILVA, L. L. CEVIC: a violência denunciada. 2005. Dissertação (Mestrado) - Programa de PósGraduação em Saúde Pública, Universidade Federal de Santa Catarina, Florianópolis.

TOSCANO, G.; WEBER. W. Violence in work place. Washington, D.C: Bureau of Labor Statistics, 1995. Available in: http://www.cdc.gov/niosh/docs/96-100/introdu ction.html/. Access in: 22 Nov 2019.

VASCONCELLOS, I. R. R.; GRIEP, R. H.; LISBOA, M. T. L.; ROTENBERG, L. Violence in daily hospital nursing work. Acta Paul Enferm. 2012; 25(spe2). 